\title{
Limits on Neutrino-Mixing from the Internal Bremsstrahlung Spectrum of ${ }^{125}$ I
}

\author{
M. J. G. Borge, ${ }^{1}$ A. De Rújula, ${ }^{1}$ P. G. Hansen, ${ }^{2}$ B. Jonson, ${ }^{3}$ G. Nyman,${ }^{3}$ H. L. Ravn, ${ }^{1}$ K. Riisager,${ }^{2}$ and the \\ ISOLDE Collaboration
}

${ }^{1}$ CERN, CH-1211 Geneva 23, Switzerland; ${ }^{2}$ Institute of Physics, University of Aarhus, DK-8000 Århus C, Denmark; ${ }^{3}$ Department of Physics, CTH, S-41296 Göteborg, Sweden.

Received June 10, 1986; accepted July 25, 1986

\begin{abstract}
The massive antineutrino recently reported by Simpson in an experiment on tritium decay has not been confirmed by experiments on ${ }^{35} \mathrm{~S}$ decay. As an independent check, we report here the negative result of a search for the corresponding antiparticle (a massive neutrino) using the decay of ${ }^{125} \mathrm{I}$. As a by-product, we give accurate data on internal-bremsstrahlung spectra which we have followed over close to four decades in intensity, and we report a new way of measuring its E1/M1 ratio. The experiment provides a new and very accurate value for the ${ }^{125} \mathrm{I}-{ }^{125} \mathrm{Te}$ mass difference of $186.1 \pm 0.3 \mathrm{keV}$.
\end{abstract}

\section{Introduction}

Whether neutrinos are massive or not is not known. This question has given rise to much activity in recent years, partly due to the published indications [1] of an electron antineutrino mass around $35 \mathrm{eV}$. While this result has been questioned $[2,3]$, several experiments now seem on the verge of giving improved limits, as can be seen from a recent overview [3] of the field. In the following, we shall be concerned with a phenomenon that may occur if neutrinos are massive, namely neutrino-mixing. This takes place if the "weak" eigenstates $v_{\mathrm{e}}, v_{\mu}$ and $v_{\tau}$ are not at the same time mass eigenstates, so that in weak processes, here nuclear beta decay or electron-capture decay, the neutrinos will have two or more mass components. Searches for admixtures of heavy neutrinos in beta decay have been performed [4-10] with tritium, ${ }^{64} \mathrm{Cu}$, and ${ }^{35} \mathrm{~S}$. A non-zero result was reported by Simpson [5], who interpreted an upbend below $1.5 \mathrm{keV}$ electron energy in the tritium decay as evidence for a $2-4 \%$ component of a $17.1 \mathrm{keV}$ neutrino. There have been some attempts [11-12] at alternative explanations of these data; most likely [12], the effect can be attributed to neglect of atomic corrections. Independent checks performed [7-10] on ${ }^{35} \mathrm{~S}$ provide limits that disagree with Simpson's interpretation, but Simpson has recently argued [3] that not all of the experiments were correctly analyzed, and that some of them may even favour the existence of an $m_{v} \sim 17 \mathrm{keV}$ component of the electron neutrino. Rather than entering this controversy here, we provide our own independent piece to the puzzle.

The $\mathrm{e}^{-}$beta-decay experiments probe the mass of the antineutrino. In order to determine the mass of the corresponding antiparticle, one must study positron spectra or, better, spectra of internal bremsstrahlung (IB). This latter approach is interesting for two reasons, viz. (i) it provides a test of CPT for neutrinos, and (ii) under favourable circumstances, it could provide a more sensitive method than $\mathrm{e}^{-}$ decay. De Rújula [13] has shown that this may be the case if the $Q$ value is resonant with one of the s-electron binding energies. In the following is described the use of the IB technique in a search for heavy neutrinos and, as a byproduct, a new way of determining the relative intensities of M1- and E1-IB is reported. The case selected for this experiment is ${ }^{125} \mathrm{I}$, which decays exclusively by electron capture by a relatively fast allowed transition to the first-excited level (at $35.5 \mathrm{keV}$ ) of ${ }^{125} \mathrm{Te}$, as shown in the inset in Fig. 4.

\section{The experiment}

\subsection{Source preparation and set-up}

The source of ${ }^{125} \mathrm{I}$ was obtained as decay product of ${ }^{125} \mathrm{Cs}$. Cesium nuclei were produced by spallation of a molten lanthanum target with a $1.5 \mu \mathrm{A}$ beam of $600 \mathrm{MeV}$ protons from the CERN synchro-cyclotron and subsequently mass separated in the ISOLDE on-line isotope-separator facility. The $1.8 \mathrm{nA}^{125} \mathrm{Cs}^{+}$beam was collected in Al foils, producing a source of $2.1 \times 10^{15}$ atoms. Small contaminations of the neighbouring masses were present, most noticeable about $10^{7}$ atoms of ${ }^{127} \mathrm{Xe}$. The sources were dissolved and purified radiochemically. After addition of $10 \mathrm{mg}$ I carrier, standard radiochemical procedures [14] based on $\mathrm{CCl}_{4}$ extractions and AgI precipitations were applied. After the chemical separation the AgI was sealed in a polyethylene tube. No traces of ${ }^{127} \mathrm{Xe}$ in the final source could be seen above the background. At the start of the data-taking, the source contained less than $10^{5}$ atoms of ${ }^{127} \mathrm{Xe}$.

For the main part of the measurement, we used a set-up with two intrinsic Ge planar detectors, both of thickness $7 \mathrm{~mm}$ and area $200 \mathrm{~mm}^{2}$. To reduce the count rate of the $\gamma$-ray and the X-rays from ${ }^{125} \mathrm{Te}$, absorber foils were placed between the source and the detectors, in the final set-up approximately $0.2 \mathrm{~mm} \mathrm{Ag}, 0.075 \mathrm{~mm} \mathrm{Mo}$, and $0.3 \mathrm{~mm} \mathrm{Cu}$. The source was sandwiched between the two detectors, which were shielded with copper and cadmium foils, the entire set-up being surrounded by a $20 \times 20 \times 20 \mathrm{~cm}^{3}$ box filled with lead shots. The singles data from both detectors were stored on magnetic tape. The total measuring time with the final set-up was 55 days.

\subsection{Calibration and data reduction}

Measurements of the background were performed intermittently through the counting period, and no major structures were seen. Standard sources of ${ }^{133} \mathrm{Ba},{ }^{241} \mathrm{Am}$, and ${ }^{57} \mathrm{Co}$ were used for the calibration measurements, which were repeated regularly to check the stability of the set-up. The system was slightly non-linear in energy (variations of $1 \mathrm{keV}$ with total range $400 \mathrm{keV}$ ); this was corrected for, so that the resulting energy calibration is good within $\sim 0.1 \mathrm{keV}$. Long- 
term fluctuations in the amplification of up to $1 \%$ were seen (probably stemming from the electronics). The data were corrected for these fluctuations, giving a final uncertainty of the energies in the IB region of less than $0.2 \mathrm{keV}$.

To test the differential linearity of the electronics we also measured the flat part of the high energy $\beta^{-}$spectrum from a source of ${ }^{106} \mathrm{Ru}$ with a plastic scintillator. No periodic structures or irregular jumps were observed. We therefore conclude that our set-up can detect local structures at intensities of relative order $10^{-3}$.

To correct for the absorption in the foils and the efficiency of the detector, the absorption coefficients of the tables of Veigele [15] and the detector data sheets from the manufacturer were used. The total detection efficiency (including the effect of the foils) was checked with the calibration sources and found to agree with the calculated values within the uncertainty of a few per cent. In doing this, we had to correct the absorption coefficients for the finite solid angle $(5-10 \%)$ of detection.

The main noteworthy feature of the detector response function at these photon energies is the backscatter peak (created by photons Compton-scattered from the surroundings into the detector), which here lies fairly close to the main peak. Its magnitude, found from calibration runs, was $2-10 \%$ of the full energy peak in the IB region. Pile-up was found to be important due to the relatively large shaping time used ( $6 \mu \mathrm{s}$ on the amplifiers), giving a contribution of order of $1 \%$ at $100 \mathrm{keV}$ and of $5 \%$ at $130 \mathrm{keV}$. From the ${ }^{57} \mathrm{Co}$ runs, the pile-up spectrum was seen to consist of a peak at the summed energy with an intensity that could be reproduced in a calculation, assuming an effective pile-up time of $2 \tau=2.4 \mu \mathrm{s}$. In addition, there was a fairly constant plateau down to the main peak, which required $2 \tau \sim 20 \mu$ s with large uncertainty. This agreed well with the pulse lengths, as seen on an oscilloscope.

The energy resolution of the detectors was $400-600 \mathrm{eV}$ in the range studied, but played no role in the analysis as the recorded spectra were converted to give linear spectra with $1 \mathrm{keV}$ per channel and then added. For a search for heavy neutrinos such a bin size is sufficient. Background was subtracted, and the spectra were unfolded on the basis of a simplified detector response function consisting of a $\delta$-function at the full energy $(77 \%)$ and a constant plateau extending down to zero $(23 \%)$. As the intensity is rapidly decreasing for increasing energy, and as we are mainly interested in the spectral endpoint, this is more than adequate for our purpose. We note that there was no higher energy lines capable of giving Compton edges or back-scatter peaks in the IB region. Small traces of the nuclei ${ }^{172} \mathrm{Hf}$ and ${ }^{173} \mathrm{Lu}$, corresponding to $(2-3) \times 10^{7}$ atoms of each isotope, were found in the spectra. They both give lines in the region $80-140 \mathrm{keV}$ that is of special interest in the IB spectrum. The amounts of these contaminations were determined from their higher energy lines, and the calculated impurity lines in the region of interest were subtracted from the spectra with their intensity suitably corrected for total detection efficiency. The spectra were corrected for pile-up with the $2 \tau=2.4 \mu \mathrm{s}$ for the summed energy peak found above; in view of the large uncertainty on the "plateau correction", we varied the $2 \tau$ for this part and used this and a remaining constant background to get a clean spectral endpoint. The pile-up plateau correction found in this way was always of the order indicated by the

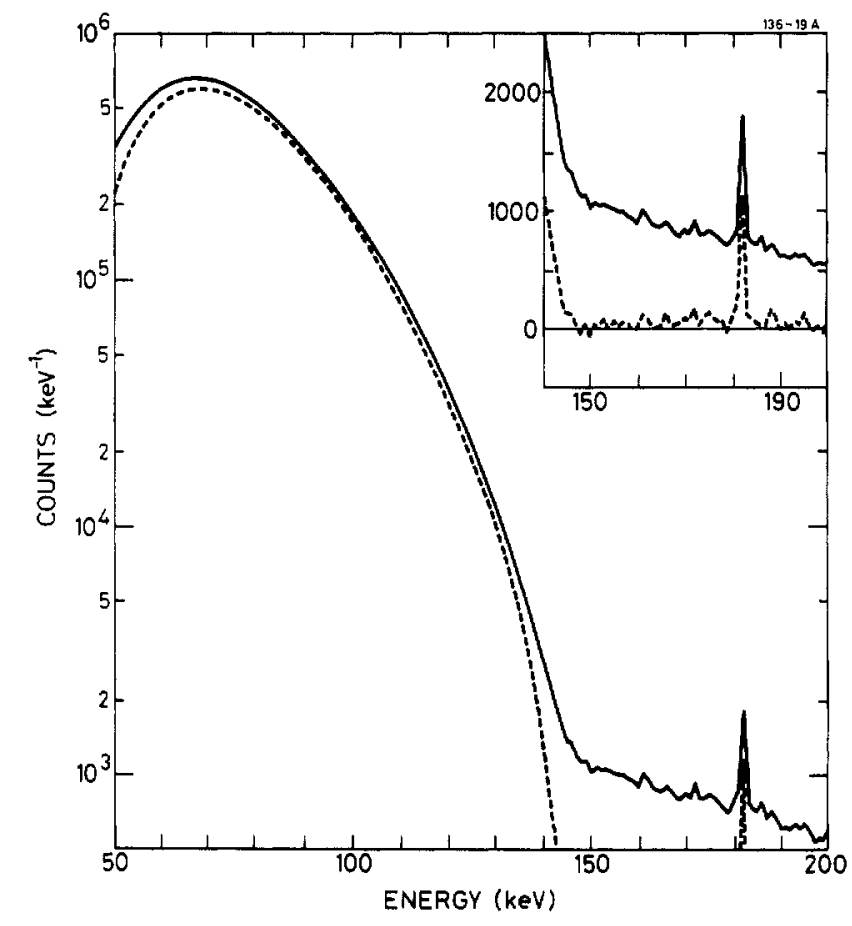

Fig. 1. The raw data (solid line) and the same data corrected for detector response, pile-up, and background (dashed line) are shown as a function of photon energy on a logarithmic scale. The inset displays the endpoint on a linear intensity scale (same energy scale) to bring out the endpoint behaviour more clearly. The peak at $182 \mathrm{keV}$ stems from ${ }^{172} \mathrm{Hf}$. The data are taken from the second detector and represent 9.4 days of data-taking.

${ }^{57} \mathrm{Co}$ runs. This final correction was unambiguous as the IB spectrum and the background were clearly separated, as illustrated in Fig. 1. We chose to apply the corrections for absorption and detector efficiency to the theoretical spectrum when fitting the data. This resulted in two final spectra, one for each detector and having roughly the same counting statistics. In the analysis these spectra were compared with a theoretical model to be discussed in the following section.

\section{Theoretical model}

\subsection{The theory of internal bremsstrahlung}

The general theory of internal bramsstrahlung was developed by Glauber and Martin [16] and a review of the theoretical and experimental work on IB has been given in [17]. The recent interest in low-energy IB stems from the observation by De Rújula [13] that information on the mass of the electron neutrino can be found from the IB spectrum. This is analogous to the way limits on the mass of the electron antineutrino are found from the electron spectrum in tritium decay: In both cases, one studies the spectral endpoint, at which the low-energy neutrinos, if massive, must become non-relativistic. The low-energy IB is predominantly $p$-wave (E1 multipole order) and is strongly resonant [16]. The special advantage in the $p$-IB spectrum is that the resonances under favourable circumstances may give large enhancements in the intensities. These "resonances" are, in fact, nothing but the $\mathrm{X}$-rays following electron capture beta decay. The ideal case would be to have an X-ray at the IB endpoint, which implies that the $Q$ value for beta decay should equal the binding energy of an atomic $s$-state. In this case, it is possible to measure the neutrino phase-space factor for low neutrino energies by studying in detail the shape of this X-ray. Unfor- 
tunately, an exact coincidence of atomic and nuclear energies does not seem to occur for any known nucleus, although several candidates have been studied during the last years [18].

A detailed study of the low energy $p$-IB based on realistic models of atomic structure has recently been performed [19]. We refer to this work for details of the theory used in the present analysis. For explicit numerical calculations, nonrelativistic Hartree-Fock wave functions [20] and atomic binding energies [21] are needed, and the calculations then proceed similarly to model (I) of Ref. [19]. The spectral intensities can consequently be calculated with the $Q$-value and the neutrino mass as the only free parameters. If neutrino mixing occurs, the statistical weight factor $S$ (eq. (2a) of [19]) is modified to have components of intensity $c_{i}$ for mass $m_{i}$, so that$$
S=\sum_{i} c_{i} k\left(Q-B_{n p}-k\right)\left[\left(Q-B_{n p}-k\right)^{2}-m_{i}^{2} c^{4}\right]^{1 / 2},
$$

where $k$ is the photon energy and $B_{n p}$ the binding energy of an $n p$-electron.

The $Q$-value of ${ }^{125} \mathrm{I}$ is favourable for a search for a neutrino with mass $15-30 \mathrm{keV}$, and very strong sources can be made of this activity. The high $Q$ value also implies that $s$-IB will contribute as well. The intensity can be calculated from the expression given by Glauber and Martin [16], here in the notation of [19],

$\frac{1}{\Sigma \omega} \frac{\mathrm{d} \omega_{n s}}{\mathrm{~d} k}=\frac{e^{2}}{\pi m^{2} \hbar c^{5}} \frac{\left|\psi_{n s}(0)\right|^{2} S}{\sum_{n^{\prime} l}\left|\psi_{n^{\prime} l}(0)\right|^{2}\left(Q-B_{n^{\prime} l}\right)^{2}}$,

where $\psi_{n l}(0)$ is the value of the wave function at the origin, and $S$ is in anlogy with eq. (1) a statistical factor. Note that by use of realistic $\psi_{n l}(0)$ values, screening is taken approximately into account [17].

\subsection{Parameters}

In the analysis we made the simplifying assumption that only two neutrino masses enter in eq. (1), with the largest component having a negligible mass (i.e., $m_{1}=0, c_{1}=1-c_{2}$, $c_{i}=0$ for $i \geqslant 3$ ). We thus have three parameters in the theoretical spectrum, namely $Q, m_{2}$ and $c_{2}$. As the nonrelativistic theory is known $[17,19]$ to reproduce the shapes of $p$-IB and $s$-IB much more accurately than the absolute magnitudes, we weight the theoretical $p$-IB and $s$-IB spectra with factors of 1 and $b$, respectively, in addition to the overall normalization factor $c$. The parameter $b$ is thus a correction factor of order unity, to be determined from experiment. As the absorption and efficienty corrections (see Section 2.2) could not be determined with the same accuracy as the spectrum itself, we have taken this uncertainty into account by multiplying the theoretical spectrum (to which the absorption and efficiency corrections were applied) by a factor $\exp (\alpha(k-110 \mathrm{keV}))$, where $k$ is the photon energy and $\alpha$ an extra free parameter. This factor could also absorb inaccuracies stemming from the rough unfolding of the detector response, and as $\alpha$ turns out to be small it cannot in any way introduce local structure in the spectrum. We note that it is common to use correction factors with linear and quadratic terms $[1,7,8]$ but feel that the exponential term is easier to justify since it resembles the uncertain terms, and is certain to vary smoothly. We end up with a total of six free parameters, $Q, c, b, \alpha, m_{2}$ and $c_{2}$.

\section{Data analysis and search for a heavy neutrino}

In order to bring out clearly the problems in the determination of $m_{2}$ and $c_{2}$ from the measured spectra, we proceed here in a stepwise fashion. We attempt first to solve the problem by inspection and then proceed to more powerful and refined statistical methods.

\subsection{The shape-factor plot}

Choosing as our reference a theory with $c_{2}=0$ (no heavy neutrino), it is possible to represent the data relative to this theory, adjusted in the best possible way with four parameters. This is illustrated in Fig. 2, in which the upper part is a simulation with "pseudo data" generated in a calculation with $m_{2}=17 \mathrm{keV}, c_{2}=0.03$. It is striking that the effect of the heavy neutrino to a large extent can be absorbed by the other parameters, in this example by small changes in $b$ and $Q$ away from their "true" values. Thus, in comparing different hypotheses for $m_{2}, c_{2}$ it is essential each time to carry out an independent adjustment of the other free parameters.

Analogous problems occur, of course, in the ${ }^{35} \mathrm{~S}$ experiments [7-10]. We feel, in complete agreement with the opinions expressed by J. J. Simpson in a contribution to [3], that the limits on $c_{2}$ derived in [9] are misleading as the parameters were not fitted again under the assumption of a heavy neutrino; instead the contribution from this was simply added to the shape factor plot. The approach taken here and

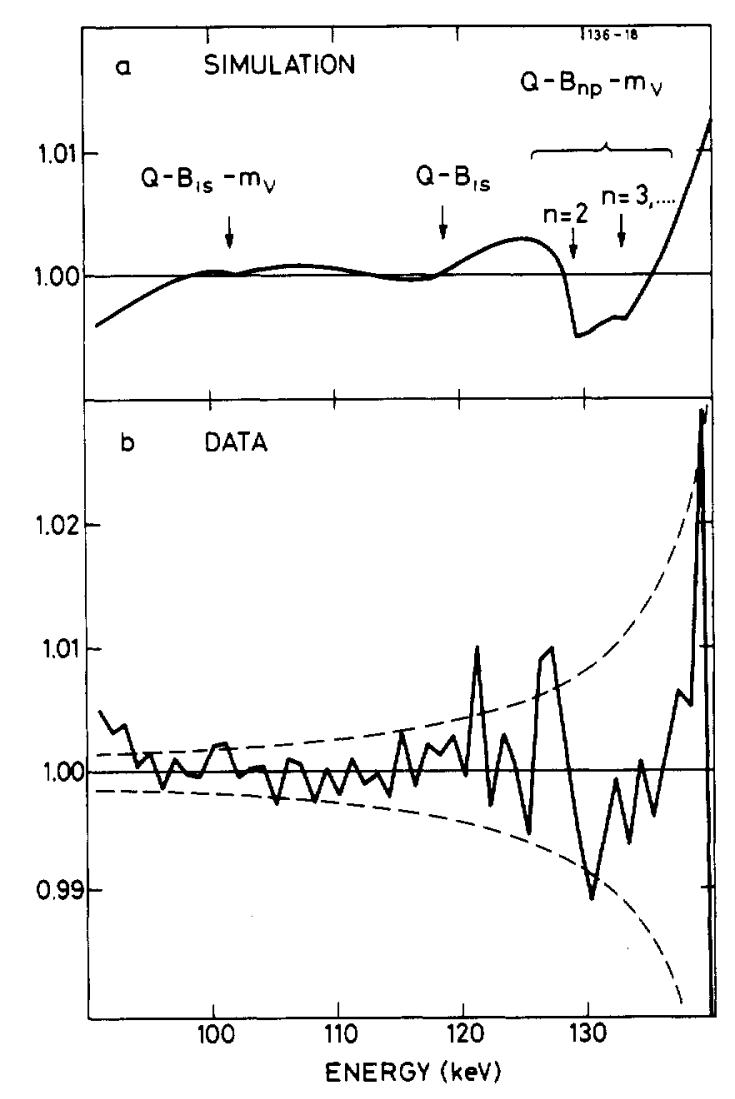

Fig. 2. Shape factor plots showing (a) a simulation, in which the constructed spectrum had a $3 \%$ component corresponding to a neutrino mass of $17 \mathrm{keV}$, and (b) the experimental results for the second detector with the dashed line indicating the statistical error at the $1 \sigma$ level. The comparison spectrum, which is divided into the data to obtain the shape factor, was in both cases calculated from a theory without a heavy neutrino and with an independent adjustment of all the free parameters. It is for this reason that the simulation shows a kink also at the $1 \mathrm{~s}$ bremsstrahlung end-point. 
also in Refs. [7] and [10] leads to much more conservative limits on $c_{2}$.

The shape factor plot of the experimental data from the second detector resembles the simulation strikingly and these data taken alone favour a heavy neutrino (Fig. 2(b)) although the effect still is consistent with a statistical fluctuation. No such effect was seen in the data from the first detector and in the combined analysis (below) of the results more restrictive limits can be put.

\subsection{Two-dimensional $\chi^{2}$ adjustment}

We have performed a combined analysis of the two data set in the interval $100-139 \mathrm{keV}$. The parameters $Q, \alpha$ and $c$ were adjusted individually for each of the two spectra, whereas common values were used for $b, m_{2}$ and $c_{2}$. In the analysis the $\chi^{2}$-function was minimized for each value of the pair $\left(m_{2}, c_{2}\right)$, thus giving 73 degrees of freedom; the resulting contour plot of $\chi^{2}$ is given in Fig. 3. In order to interpret Fig. 3 we now recall some basic elements of statistical analysis by means of likelihood ratios.

Assume a hypothesis $H_{s}$ depending on $s$ free parameters $p_{1}$, $p_{2}, \ldots, \dot{p}_{s}$. The likelihood $L\left(x_{i}, H_{s}\right)$ is proportional to the probability of having the outcomes $x_{1}, x_{2}, \ldots, x_{n}$. The $x_{i}$ are taken to be the measured values. The parameters $p_{1}$, $p_{2}, \ldots, p_{s}$ are chosen to maximize $L$, or, more often, a quantity $S=\ln L$, occasionally called support. It is well known that this procedure is identical to a minimization of $\chi^{2}$ for the case of a normally distributed variable.

For a fixed set $x_{i}$ of measured values we now wish to compare the hypothesis $H_{s}$ with one $H_{s+i}$ that has $t$ additional free parameters. To judge the value of this increase in the parameter space we consider the resulting increase in support or, equivalently, the decrease in $\chi^{2}$ at the new minimum $-\Delta \chi^{2}=\chi^{2}(n-s)-\chi^{2}(n-s-t)$. It can be shown [23] that if the zero hypothesis holds, that is if the $t$ new parameters bring no essential improvement in the fit, the quantity $-\Delta \chi^{2}$ is distributed according to a $\chi^{2}$ distribution with $t$ degrees of freedom.

We now return to Fig. 3 and note that the value of $\chi^{2}$ on the $x$-axis of 69.2 is in comfortable agreement with the expected value of 73 . This is a confirmation of our basic hypothesis that apart from small deviations in the absolute values the standard theory of internal bremsstrahlung works well. We consider now the effect of adding two more theoretical parameters, $m_{2}$ and $c_{2}$; there are then 71 degrees of freedom away from the $x$-axis. Even if $m_{2}$ and $c_{2}$ should turn out to be unnecessary, we still expect from the arguments given above that the minimum in $\chi^{2}$ will decrease by two units, that is to 67.2. For two degrees of freedom there is a $10 \%$ probability that the decrease will exceed 4.6 units, corresponding to 64.6 for $\chi^{2}$. A minimum deeper than this may be taken as an indication for a second neutrino at the $90 \%$ confidence level.

In the part of the $\left(m_{2}, c_{2}\right)$ subspace examined there are three minima, two local ones at $\chi^{2}=65.2$ and 65.8 , and one, extending along the axis for slightly negative $c_{2}$, also around 66. By the criterion developed above, neither of these are significant. The $90 \%$ confidence level contour lies at approximately 70 , i.e., 4.6 units higher than the lowest minimum. This contour is open, that is it includes zero; upper limits of $c_{2}$ well below $1 \%$ are obtained for $m_{2}$ between 17 and $35 \mathrm{keV}$.

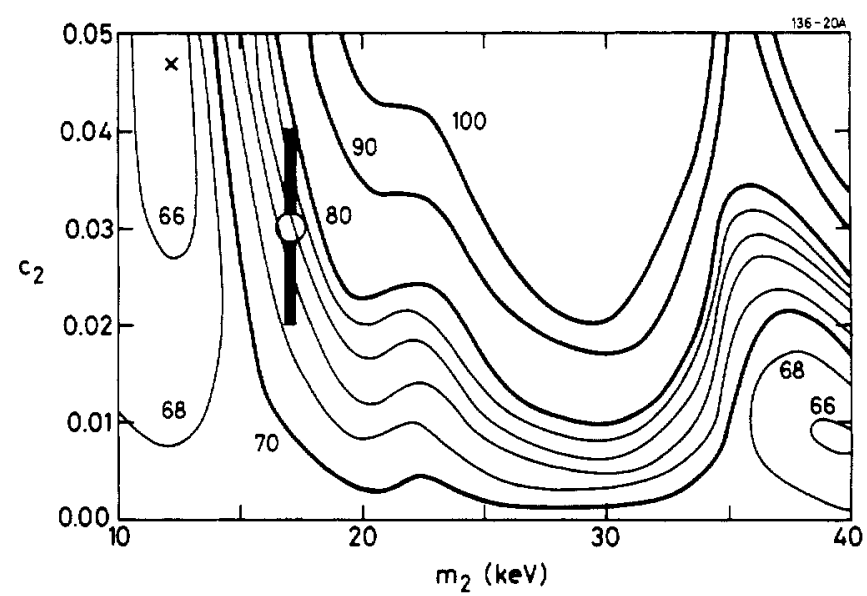

Fig. 3. Contour plot of $\gamma^{2}$ as a function of the neutrino mass $m_{2}$ and the mixing parameter $c_{2}$. The curves are labelled by the value of $\chi^{2}$. The absolute minimum value of $\chi^{2}$ is 65.2 and is marked with a cross, and there are 71 degrees of freedom when all six parameters are adjusted. The $90 \%$ confidence limit $[22,23]$ (see text) lies near the contour with $\chi^{2}=70$, and the permitted region extends along the axis and includes all local minima. The $99.5 \%$ confidence level lies near the $\chi^{2}=76$ contour. The loss of sensitivity at $m_{2}=34 \mathrm{keV}$ (a few $\mathrm{keV}$ above the $1 s$-binding energy) arises when the $1 s$-IB endpoint and the neutrino-mass signal from the $n p$-IB spectra coincide (see Fig. 2(a)). The point with error bars is Simpson's [5] result from the tritium experiment.

Simpson's $17.1 \mathrm{keV}$ neutrino with a $c_{2}$ of $0.02-0.04$ is also shown in Fig. 3, from which it is seen that the corresponding antineutrino would have a $\chi^{2}$ of 72.6 and 79 for the ends of the $c_{2}$ interval. This means that the exclusion is at confidence levels of $98 \%$ and $99.9 \%$, respectively.

\section{Experimental results}

We now summarize the conclusions drawn from the analysis discussed in Section 4.

\subsection{The parameters $Q$ and $b$}

The only other reported measurement of the IB-spectrum of ${ }^{125}$ I gave a $Q$-value [24] for the decay to the first excited state of ${ }^{125} \mathrm{Te}$ of $Q=141.5 \pm 2 \mathrm{keV}$. From the fits with $c_{2}=0$, we find from the first detector $150.35 \pm 0.15 \mathrm{keV}$ and from the second detector $150.88 \pm 0.16 \mathrm{keV}$. Taking into account the uncertainty due to the allowed range of $c_{2}$ and $m_{2}$ values, we get instead $150.4 \pm 0.4 \mathrm{keV}$ and $150.8 \pm 0.4 \mathrm{keV}$. Adding the uncertainty from the energy calibration $(0.2 \mathrm{keV})$ in quadrature and taking the mean value of our two measurements gives the final $Q=150.6 \pm 0.3 \mathrm{keV}$, which implies a total $Q$-value for the decay of ${ }^{125} \mathrm{I}$ to the ground state of ${ }^{125} \mathrm{Te}$ of $186.1 \pm 0.3 \mathrm{keV}$. This is in disagreement with [24] but does not conflict with other known data [25] on ${ }^{125} \mathrm{I}$.

For the $b$-parameter, which is the empirical correction factor for the relative $s$-IB intensity, we find similarly for the two detector $0.38 \pm 0.04$ and $0.49 \pm 0.02$ when setting $c_{2}=0$, and we find $0.35 \pm 0.10$ and $0.49 \pm 0.06$ when $c_{2}$ is allowed to vary. The systematic uncertainty associated with the absorption and efficiency correction is hard to calculate and could very well be different for the two detectors. We believe that a safe estimate is $b=0.45 \pm 0.15$. For a given energy the intensity of $p$-IB is always much larger than the intensity of $s$-IB, the difference being smallest at photon energies near $100 \mathrm{keV}$, here amounting to a factor of four. That we nevertheless are able to make a rather precise deter- 
mination of $b$ indicates that the IB spectral shapes are given very well by the theory over large intervals. The $\alpha$ parameters found for the two spectra are around $-3 \times 10^{-3}$ and $-4.5 \times 10^{-3}$ respectively, so that the correction factor $\exp (\alpha(k-110 \mathrm{keV}))$ gives at most a $10 \%$ change over the energy regions used in the fits. (The normalization constants also agree with the value expected from the estimated number of atoms in the source.)

\subsection{Absolute normalization of intensity}

A measurement with the source at a distance of $40 \mathrm{~cm}$ from the detectors (to reduce the count rate) and without any absorber was used to find the absolute activity of the source. From the known decay scheme [26] and the number of $\mathrm{Te} \mathrm{K}$ $\mathrm{X}$-rays measured, corrected for self-absorption in the source, the activity can be found. The intensity of the $\mathrm{Ag} \mathrm{X}$-rays seen in the spectrum was used to estimate the amount of selfabsorption in the source, which consists of AgI. The total number of atoms in the source found in this way agreed with the estimated strength (Section 2.1). Immediately before this, a short experiment in close geometry was done with the second detector using $0.1 \mathrm{~mm} \mathrm{Ag}$ and $0.3 \mathrm{~mm} \mathrm{Cu}$ as absorber and a shaping time of $3 \mu \mathrm{s}$. The solid angle of this detector was determined to be $11 \pm 1 \%$ by comparing runs with a ${ }^{133} \mathrm{Ba}$ source in different geometries and further checked with other calibration sources of known activity. With this information the experimental internal bremsstrahlung spectrum

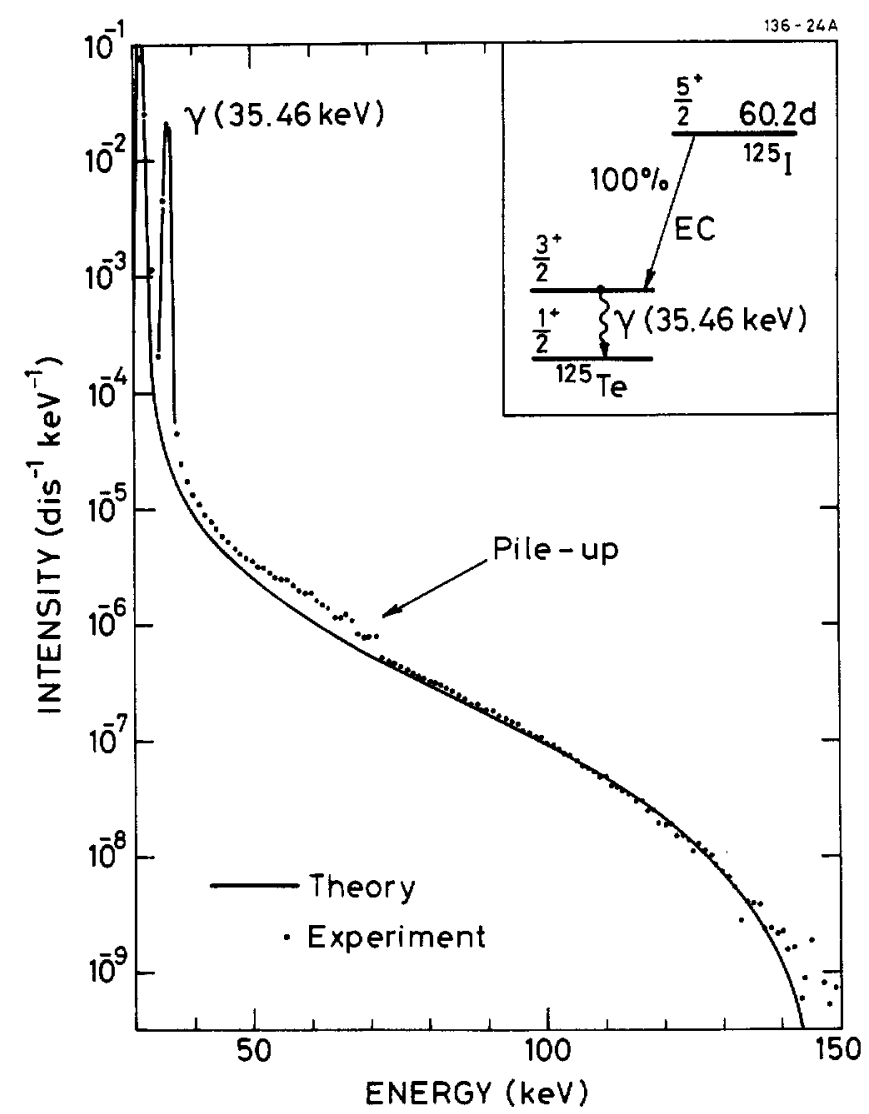

Fig. 4. The internal bremsstrahlung spectrum of ${ }^{125} \mathrm{I}$. The solid line gives the theoretical value, the dots the experimental spectrum, both on an absolute scale. The data are from the second detector and were taken with $0.1 \mathrm{~mm} \mathrm{Ag}$ and $0.3 \mathrm{~mm} \mathrm{Cu}$ absorbers and a shaping time of $3 \mu \mathrm{s}$. They are corrected for absorption and detector efficiency and unfolded for detector response. Background is subtracted, but no correction for pile-up has been attempted. The peak at $35 \mathrm{keV}$ is the $\gamma$ line stemming from the decay of the excited level in ${ }^{125} \mathrm{Te}$. The inset shows the decay scheme of ${ }^{125} \mathrm{I}$. can be displayed on an absolute scale as shown in Fig. 4. We did not attempt to correct this spectrum for pile-up, as a proper correction is difficult and as the effects of pile-up are not large. As indicated in the figure, the bumps around $65 \mathrm{keV}$ are due to pile-up. At the end of the experiment, this determination of the absolute intensity of the IB was repeated, now with the activity spread out on a larger area in order to reduce the self-absorption in the source. The two determinations of absolute intensity agreed to within $10 \%$.

Figure 4 also gives the theoretical IB-curve, where $Q=$ $150.5 \mathrm{keV}$ and $b=0.5$ was used. The spectra can be compared from the $K_{\beta}$ line of Te at $31 \mathrm{keV}$ up to $140 \mathrm{keV}$, the only interfering structure being the $\gamma$-ray at $35 \mathrm{keV}$ and the pile-up peaks around $65 \mathrm{keV}$. The shape is reproduced very well. As was clear from the shape-factor plots, local structures are reproduced within $10^{-3}$. From the region above the pile-up, we conclude that the global shape is reproduced within at least $10 \%$, allowing for systematic errors in the absorption correction. The theoretical and experimental intensities agree within the uncertainty on the experimental spectrum, which is given mainly by the uncertainty of the normalization. We estimate this to be $30 \%$. The $p$-IB is thus given absolutely by theory, whereas the $s$-IB theoretical intensity is a factor of two too high (as $b=0.5$ was used).

\section{Conclusions}

As can be seen from Fig. 3 and the discussion in Section 4.2, our experiment excludes a $17 \mathrm{keV}$ neutrino of $2-4 \%$ [5] at confidence levels of $98 \%$ and $99.9 \%$ for the ends of the interval. It supports the results of the ${ }^{35} \mathrm{~S}$ measurements [7-10], which exclude the corresponding antineutrino. The IB experiments are more complex than those based on $\beta^{-}$decay, and our limits are necessarily less restrictive than those of Refs. [7-10]. We have extended the limits of Schreckenbach et al. [6] for the electron neutrino from $50 \mathrm{keV}$ down to $10 \mathrm{keV}$. There is to the best of our knowledge no published experiment covering the region below $10 \mathrm{keV}$ based on nuclear beta decay (although other types of experiments cover this region [27]).

The internal bremsstrahlung spectrum of Fig. 4, as well as the shape-factor plot of Fig. 2, show that the shape of the IB spectrum is given very accurately by the present theory. The absolute magnitudes for both $p$-IB and $s$-IB are given within a factor of two, in full accordance with the conclusions of [19]. The present experiment, with its larger sensitivity, only strengthens our confidence in the theory of the shape of low-energy internal bremsstrahlung near resonances. Our sensitivity has furthermore allowed us to determine the E1/M1 ratio of the IB directly from the singles spectrum.

We intend to continue work on IB as it provides a valuable independent check on the mainstream experiments that use tritium decay. The early hopes [13] on IB as a possible new way in neutrino-mass measurements were justified, but so far Nature has failed to provide the resonant case that could permit a major step ahead for IB experiments. Calorimetric measurements of electron-capture decays [28] are, however, still a source of hope.

\section{Acknowledgements}

We would like to thank J. J. Simpson for interesting discussions. One of us (KR) was supported by the Danish Natural Science Research Council. 


\section{References}

1. Lubimov, V. A., Novikov, E. G., Nozik, V. Z., Tretjakov, E. F., and Kosik, V. S., Phys. Lett. 94B, 266 (1980); Boris, S., Golutvin, A., Laptin, L., Lubimov, V., Nagovizin, V., Novikov, E., Nozik, V., Soloshenko, V., Tihomirov, I., and Tretjakov, E., Phys. Lett. 1598, 217 (1985).

2. Simpson, J. J., Phys. Rev. D30, 1110 (1984); Bennett, C. L., McDonald, A. B., Springer, P. T., Chupp, T. E., and Tate, M. L., Phys. Rev. C31, 197 (1985); Bergkvist, K. E., Phys. Lett. 154B, 224 (1985); Bergkvist, K. E., Phys. Lett. 159B, 408 (1985).

3. Proc. Sixth Moriond Workshop on Massive Neutrinos (Edited by J. Tran Thanh Van), Editions Frontieres, France (1986).

4. Simpson, J. J., Phys. Rev. D24, 2971 (1981).

5. Simpson, J. J., Phys. Rev. Lett. 54, 1891 (1985).

6. Schreckenbach, K., Colvin, G., and von Feilitzsch, F., Phys. Lett. 129B, 265 (1983).

7. Altzitzoglou, T., Calaprice, F., Dewey, M., Lowry, M., Piilonen, L., Brorson, J., Hagen, S., and Loeser, F., Phys. Rev. Lett. 55, 799 (1985).

8. Apalikov, A., Boris, S., Golutvin, A., Laptin, L., Lubimov, V., Myasoedov, N., Nagovizin, V., Novikov, E., Nozik, V., Soloshenko, V., Tihomirov, I., and Tretjakov, E., Pisma Zh. Eksp. Teor. Fiz. 42, 233 (1985) [JETP Lett. 42, 289 (1985)]

9. Ohi, T., Nakajima, M., Tamura, M., Matsuzaki, T., Yamazaki, T., Hashimoto, O,.., and Hayano, R. S., Phys. Lett. 160B, 322 (1985); Datar, V. M., Baba, C. V. K., Bhattacherjee, S. K., Bhuinya, C. R., and Roy, Amit, Nature 318, 547 (1985).

10. Markey, J. and Boehm, F., Phys. Rev. C32, 2215 (1985).

11. Haxton, W. C., Phys. Rev. Lett. 55, 807 (1985); Kalbfleisch, G. R. and Milton, K. A., Phys. Rev. Lett. 55, 2225 (1985); Drukarev, E. G. and Strikman, M. I., Pisma Zh. Eksp. Teor. Fiz. 42, 472 (1985) [JETP Lett. 42, 586 (1985)].

12. Lindhard, J. and Hansen, P. G., Phys. Rev. Lett. 57, 956 (1986).

13. De Rújula, A., Nucl. Phys. B188, 414 (1981).

14. Kleinberg, J. and Cowan, G. A., National Academy of Sciences, Nuclear Science Series NAS-NS 3005 (1960).

15. Veigele, W. J., Atomic Data Tables 5, 51 (1973).

16. Glauber, R. J. and Martin, P. C., Phys. Rev. 104, 158 (1956); Martin, P. C. and Glauber, R. J., Phys. Rev. 109, 1307 (1958).

17. Bambynek, W., Behrens, H., Chen, M. H., Crasemann, B.
Fitzpatrick, M. L., Ledingham, K. W. D., Genz, H., Mutterer, M., and Intemann, R. L., Rev. Mod. Phys. 49, 77 (1977).

18. Andersen, J. U, Beyer, G. J., Charpak, G., De Rújula, A., Elbek, B., Gustafsson, H. A., Hansen, P. G., Jonson, B., Knudsen, P., Lægsgaard, E., Pedersen, J., and Ravn, H. L., Phys. Lett. 113B, 72 (1982); Jonson, B., Andersen, J. U., Beyer, C. J., Charpak, G., De Rújula, A., Elbeck, B., Gustafsson, H. A., Hansen, P. G., Knudsen, P., Lægsgaard, E., Pedersen, J., and Ravn, H. L., Nucl, Phys. A396, 479 (1983); Bennett, C. L., Hallin, A. L., Naumann, R. A., Springer, P. T., Witherell, M. S., Chrien, R. E., Baisden, P. A., and Sisson, D. H., Phys. Lett. 107B, 19 (1981); Baisden, P. A., Sisson, D. H., Niemeyer, S., Hudson, B., Bennett, C. L., and Naumann, R. A., Phys. Rev. C28, 337 (1983); Yasumi, S., Rajasekaran, G., Ando, M., Ochiai, F., Ikeda, H., Ohta, T., Stefan, P. M., Maruyama, M., Hashimoto, N., Fujioka, M., Ishii, K., Shinozuka, T., Sera, K., Omori, T., Izawa, G., Yagi, M., Masumoto, K., and Shima, K., Phys. Lett. 122B, 461 (1983); Beyer, G. J., De Rújula, A., von Dincklage, R.-D., Gustafsson, H. A., Hansen, P. G., Hoff, P., Jonson, B., Ravn, H. L., and Riisager, K., Nucl. Phys. A408, 87 (1983); Raghavan, R. S., Phys. Rev. Lett. 51, 975 (1983); von Dincklage, R.-D., Hay, H. J., and Ravn, H. L., Nucl. Phys. A445, 113 (1985).

19. Riisager, K., De Rújula, A., Hansen, P. G., Jonson, B., and Ravn, H, L, Physica Scripta 31, 321 (1985).

20. Clementi, E. and Roetti, C., Atom. Nucl. Data Tables 14, 177 (1974).

21. Larkins, F. P., Atom. Nucl. Data Tables 20, 311 (1977).

22. Eadie, W. T., Drijard, D., James, F. E., Roos, M., and Sadoulet, B., Statistical Methods in Experimental Physics, North-Holland, Amsterdam (1971); James, F., CERN Computer Centre Program Library, D506 Supplement to long-write-up (1978).

23. Kendall, M. G. and Stuart, A., The Advanced Theory of Statistics, Vol. 2, 3rd ed., pp. 240-241, Griffin, London (1973).

24. Gopinathan, K. P. and Rubinson, W., Bull. Am. Phys. Soc. 13, 1452 (1968).

25. Wapstra, A. H., Audi, G., and Hoekstra, R., Nucl. Phys. A432, 185 (1985).

26. Lederer, C. M., Shirley, V. S., Browne, E., Dairiki, J. M., Doebler, R. E., Shihab-Eldin, A. A., Jardine, L. J., Tuli, J. K., and Buyrn, A. B., Table of Isotopes, 7 th edition, Wiley, New York (1978).

27. Blümer, H. and Kleinknecht, K., Phys. Lett. 161B, 407 (1985).

28. De Rújula, A. and Lusignoli, M., Phys. Lett. 181B, 429 (1982). 